\title{
Fatores que influenciam os enfermeiros a utilizarem a atividade física na assistência a pacientes psiquiátricos
}

\author{
FACTORS WHICH INFLUENCE NURSES TO PROMOTE PHYSICALACTIVITIES AMONG \\ PSYCHIATRIC PATIENTS \\ FACTORES QUE INFLUYEN EN LOS ENFERMEROS A USAR LAACTIVIDAD FÍSICA EN \\ LAASISTENCIAAPACIENTES PSIQUIÁTRICOS
}

Elda de Oliveira ${ }^{1}$, Marli Alves Rolim²

\section{RESUMO}

O estudo trata da importância da promoção de atividades fisicas junto a pacientes psiquiátricos e os objetivos foram identificar o potencial de mudança de

comportamento e as crenças dos enfermeiros acerca dos beneficios das atividades físicas para os pacientes psiquiátricos, caracterizar como as atividades físicas são desenvolvidas no hospital psiquiátrico e levantar os fatores que os influenciam a promoverem atividades físicas. Os 14 enfermeiros entrevistados acreditam nos beneficios dos exercícios físicos, mas apenas 9 desenvolvem atividades. Baseados no questionário de Matsudo $^{(I)}$, chegou-se à conclusão que $50 \%$ estão no estágio de ação, 42,9\% são contemplativos e apenas 7,1\% estão na fase précontemplativa.

\begin{abstract}
This study addresses the importance of promoting physical activities among psychiatric patients and its goals were to identify the changing behavior potential of the nurses as well as to identify the nurse staff beliefs regarding the benefits of physical activity with the patients, to characterize the way in which these activities are managed inside the psychiatric hospital and finally, to identify the factors which influence the promotion of these activities in the mentioned environment. The fourteen nurses interviewed believe in the benefits of physical exercise, but only nine of them do training. Based on the Matsudo questionnaire ${ }^{(8)}$, we have noticed that $50 \%$ of them are in the action stage, $42.9 \%$ are in contemplative stage and only $7.1 \%$ are in the pre-contemplative stage.
\end{abstract}

\section{KEYWORDS}

Nurse. Physical Activity.

Nursing Care.

Psychiatric nursing.

\section{RESUMEN}

El presente estudio trata de la importancia de la promoción de actividades fisicas para pacientes psiquiátricos siendo el enfermero el miembro, del equipo multiprofesional, que puede influir en tal comportamiento. Los objetivos de la investigación fueron identificar el potencial de cambio de comportamiento y las creencias de los enfermeros respecto a los beneficios de las actividades físicas, caracterizar el desarrollo de las actividades físicas en el hospital psiquiátrico y determinar los factores que los inducen a promover actividades fisicas. Fueron entrevistados 14 enfermeros y todos acreditaban en los beneficios de los ejercicios fisicos para los pacientes, sin embargo sólo 9 desarrollan actividades fisicas con los mismos. Basados en el cuestionario de Matsudo ${ }^{(8)}$, encontramos que $50 \%$ están en el campo de acción, 42,9\% son contemplativos y sólo 7,1\% están en la fase precontemplativa.

\section{PALABRAS-CLAVE}

Enfermera. Actividad fisica. Atencion de enfermeria. Enfermería psiquiátrica.
1 Aluna de Iniciação Científica financiada pela FAPESP. cosdam@osite.com.br 2 Professora Doutora, EEUSP. Orientadora deste estudo. minie@usp.br
Enfermeira. Atividade fisica. Cuidados de enfermagem.

Enfermagem psiquiátrica. 


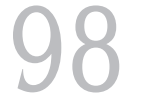

Rev Esc Enferm USP 2003; 37(3): 97-105.

\section{INTRODUÇÃO}

Recentes investigações sugerem que os adultos não participam o suficiente de atividades físicas. A inatividade tem sido uma preocupação constante daqueles que estão envolvidos, direta ou indiretamente, com a saúde, quer seja ela de ordem física ou mental ${ }^{(1-3)}$.

A falta de atividade física regular constitui-se em um dos quatros maiores fatores de risco para doenças cardíacas coronarianas, doenças estas amplamente preveníveis. A participação regular em atividade física ajuda a prevenir e reduzir os riscos associados a doenças isquêmicas, além de produzir efeitos benéficos em outras condições de saúde como a osteoporose, diabetes, hipertensão e depressão(4)

Maiores aumentos na atividade física da população dependem de mudanças no ambiente físico e social, bem como da influência de políticas nesse âmbito. Mesmo algo tão simples como caminhar depende da disponibilidade de locais seguros e atrativos para se caminhar; a atividade física precisa tornar-se não só uma escolha, mas também uma escolha fácil, consistente e agradável ${ }^{(1)}$.

No que tange à importância da atividade física para uma vida saudável, o estudo de Karnoven $^{(2)}$ ressalta que a atividade física mostrou acentuadas associações positivas como a capacidade para o trabalho, estilo de vida e bem estar. Então, além de reduzir morbidade e mortalidade, esta, aliada ao preparo físico, também promove saúde mental e social, além de aumentar a qualidade de vida das pessoas.

O significado da atividade física para uma sociedade não está limitado somente ao âmbito da saúde. Há cada vez mais evidências de que ela também tem importância para a economia, para a vida no trabalho, para a socialização e educação.

Em estudo realizado com 10 portadores de deficiência física, na sua maioria com lesão medular, verificou-se que a reabilitação e inserção em uma atividade socialmente ativa e aceita, no caso ser componente do basquete em cadeira de rodas, influenciam de forma positiva as representações sobre "Qualidade de Vida".Para as autoras é de suma importância estimular o desenvolvimento e implementação de atividades de tal natureza como de obtenção da re-significação da própria identidade e do sentido da própria vida, reconstruindo assim, os papéis sociais ${ }^{(5)}$.

Sabemos que os doentes mentais exercitam-se menos que as pessoas em geral, quer seja como decorrência da própria patologia psiquiátrica, que em alguns casos provocam lentificação psicomotora, quer seja como conseqüência dos tratamentos medicamentosos que acabam comprometendo a motricidade dos pacientes.

A avaliação da qualidade de vida geralmente é determinada pelo desempenho de um indivíduo numa variedade de domínios, tais como o estado físico, estado mental, interação social e atividades ocupacionais ${ }^{(6)}$.

Os exercícios podem ter uma função protetora frente à doença mental, pois provocam menos efeitos adversos do que algumas drogas e também apresentam baixo custo. Contudo, os exercícios físicos raramente são oferecidos como opção de tratamento ou como coadjuvante em clínicas psiquiátricas ou hospitais dia; talvez esse fato reflita a relutância em considerar a totalidade mente e corpo ${ }^{(7)}$.

Segundo Broocks et al. ${ }^{(8)}$,vários estudos com voluntários sadios têm mostrado o efeito positivo do treinamento aeróbico, persistente, em sintomas de ansiedade, na auto-estima, concentração e tolerância ao estresse. Segundo esses autores, há somente poucos estudos controlados examinando o potencial terapêutico dos exercícios em desordens psiquiátricas. Contudo, há uma boa evidência de que o exercício é efetivo em depressão moderada e nos transtornos ansiosos. O efeito terapêutico não foi correlacionado com mudanças na atividade cardiopulmonar, na maioria dos estudos. Contudo, outros mecanismos neurobiológicos e psicológicos são discutidos, os quais podem explicar a efetividade de um treinamento físico regular. Nesses estudos os autores oferecem uma variedade de sugestões de como os exercícios podem ser integrados e avaliados no tratamento de transtornos psiquiátricos.

No âmbito da enfermagem psiquiátrica, o que temos percebido é que a responsabilidade que o enfermeiro tinha em oferecer atividades terapêuticas ao paciente, passou para o terapeuta ocupacional. Contudo, na maioria dos hospitais, ocupar o paciente ainda é tarefa que cabe ao pessoal de enfermagem, 
sob a coordenação da enfermeira, havendo poucos estudos que focalizem a promoção de atividades físicas.

Dentro da perspectiva que enfoca a inatividade física como um problema de saúde coletiva, salienta-se um programa para a promoção de saúde através da atividade física. Denominado “Agita São Paulo", este programa foi desenvolvido pelo Centro de Estudos do Laboratório de Aptidão Física de São Caetano do Sul (CELAFISCS), a pedido da Secretaria de Saúde do Estado de S. Paulo.

A finalidade desse programa, em suma, é acrescentar atividade física como uma importante ferramenta para a promoção de saúde e melhora da qualidade de vida, e uma das estratégias seria atualizando as atividades dos profissionais de saúde ${ }^{(9)}$.

Nessa mesma linha de pensamento, encontra-se o estudo que aponta o comportamento pessoal das enfermeiras como elemento importante na promoção das atividades físicas. Enfermeiras que estão engajadas em exercícios físicos regulares encorajam mais a atividade física como um tratamento do que aquelas que se mostram irregularmente $\operatorname{ativas}^{(9)}$.

Com o objetivo de verificar a promoção de atividades físicas pelos profissionais de saúde e, em especial, enfermeiras assistenciais, Mackenna et al. ${ }^{(10)}$ enviaram a 846 sujeitos um questionário que avaliava os tipos de barreiras e os níveis de influência desses profissionais, bem como o estágio de mudança para a promoção de atividade e de seu comportamento pessoal. Um dos principais achados é que os profissionais em estágio de "AÇÃO", ou "MANUTENÇÃO" de mudança de sua própria atividade, promovem três vezes mais a atividade física com seus pacientes do que aqueles em outros estágios.

Diante das considerações acima e partindo do pressuposto de que as crenças dos profissionais acerca do valor terapêutico do exercício físico acaba por delinear seu maior ou menor envolvimento, ou mesmo incentivo, para essas atividades físicas a fim de torná-las, na prática, como um recurso ao tratamento dos indivíduos com sofrimento psíquico, delinearam-se como objetivos deste estudo, os que se seguem.

\section{OBJETIVOS}

- Identificar as crenças das enfermeiras acerca dos benefícios dos exercícios físicos.

- Caracterizar as atividades físicas de acordo com o tipo e a freqüência com que são desenvolvidas na unidade psiquiátrica.

- Levantar as dificuldades e facilidades enfrentadas pelas enfermeiras para promoverem as atividades físicas junto ao paciente.

- Correlacionar o diagnóstico do nível de atividade física das enfermeiras com sua prática cotidiana de promoção de atividades físicas junto aos pacientes.

\section{ABORDAGEM METODOLÓGICA}

\section{Local do estudo}

O estudo foi realizado no Instituto de Psiquiatria do Hospital das Clínicas da Faculdade de Medicina da Universidade de São Paulo, que atende a pacientes psiquiátricos, de ambos os sexos, em regime de internação e de atendimento extra-hospitalar.

\section{População}

A população estudada foi constituída por quatorze enfermeiros que atuam na referida instituição, nos turnos da manhã e da tarde.

\section{Procedimentos e Instrumento de Coleta de dados}

A coleta de dados foi realizada após prévia autorização das instâncias competentes e previamente à realização das entrevistas, os profissionais foram informados acerca da finalidade da pesquisa, do caráter sigiloso das mesmas, da possibilidade de interromperem a participação em qualquer fase, sem qualquer tipo de prejuízo. Após a aceitação em participar do estudo, assinaram o "Termo de Consentimento Livre e Esclarecido".

As entrevistas foram realizadas pela própria pesquisadora, por meio de entrevista semi-estruturada, orientada por roteiro. As entrevistas foram gravadas com a aquiescência dos enfermeiros e, posteriormente, transcritas na íntegra.

O Anexo - Instrumento de coleta de dados foi composto de três partes.
Fatores que influenciam

os enfermeiros a

utilizarem a atividade

física na assistência a pacientes psiquiátricos 
A primeira parte abrange dados referentes à identificação dos sujeitos da pesquisa.

A segunda parte refere-se ao levantamento de informações referentes às crenças dos sujeitos acerca das atividades físicas, a seus benefícios e à forma como promovem atividades físicas junto aos pacientes psiquiátricos. Esta parte compreendeu questões abertas e fechadas.

A terceira parte constituiu-se da avaliação do nível de atividade física dos enfermeiros, realizada por meio de questionário de Matsudo, adaptado dos modelos internacionais que fazem o diagnóstico do nível de atividade física e citado em documento da Secretaria do Estado da Educação de São Paulo $^{(9)}$.

\section{Análise dos dados}

Os dados obtidos a partir dos itens referentes à identificação dos sujeitos da pesquisa foram submetidos a uma análise quantitativa, posteriormente descrita em termos absolutos e percentuais. $\mathrm{O}$ mesmo processo ocorreu com os dados obtidos a partir do "Questionário para diagnóstico do nível de atividade física da população alvo. No tocante às questões abertas, a análise foi qualitativa, por meio da "Análise de Conteúdo" das expressões dos enfermeiros.

\section{RESULTADOS E DISCUSSÃO}

\section{Caracterização da população}

Participaram do estudo 14 enfermeiros, dos quais $2(14.2 \%)$ eram do sexo masculino e $12(85,8 \%)$ do sexo feminino. No tocante à faixa etária, a idade dos enfermeiros variou entre 28 e 48 anos, sendo que o grupo etário de maior freqüência foi do intervalo entre 34 a 38 anos, todos do sexo feminino, num total de 5 enfermeiras $(35,7 \%)$.

\section{Atividades fisicas: tipos, freqüência e crenças acerca de seus beneficios e maleficios.}

Quando abordados se desenvolvem atividades físicas junto aos pacientes, 9 enfermeiros responderam positivamente e 5 negativamente, sendo que dois deles, apesar de não desenvolverem atividades físicas junto com os pacientes, aproveitam as orientações que ocorrem no ambulatório para reforçar a importância das atividades físicas.
No tocante ao tipo de atividades, alguns enfermeiros citaram mais de um tipo, como por exemplo: futebol (4 citações), caminhada (2), pingue-pongue (1), basquete (1), ginástica (1), passeios externos (1), relaxamento (1) e bailes (1).

A atividade mais citada pelos enfermeiros foi o futebol. Tal acontecimento pode ser explicado pelo fato dele ser uma atividade física bastante aceita pelos brasileiros, fazendo parte do seu cotidiano e que exige pouco em termos de equipamento e orientação de algum profissional. $\mathrm{O}$ relato mostrado a seguir, pode retratar esta situação.

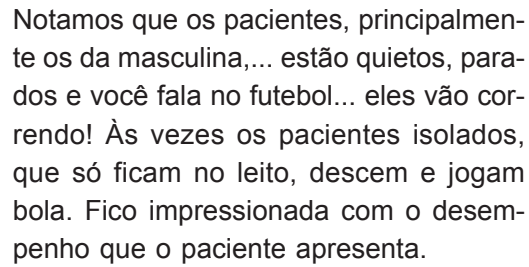

Geralmente os efeitos terapêuticos das atividades recreacionais como jogos, esportes, discussões em grupo, não são reconhecidos. Um exemplo espantoso ocorre quando um grupo de pacientes esquizofrênicos, que provavelmente nunca alcançaram a tarefa de desenvolvimento de aprendizagem de como competir e se comprometer, são bem sucedidos num time de esportes como beisebol ou futebol ${ }^{(11)}$.

Todos os enfermeiros entrevistados acreditam nos benefícios do exercício para os pacientes psiquiátricos. Tais benefícios foram categorizados a partir do seu caráter físico, psíquico e social, o que pode ser verificado no Quadro 1. 
Quadro 1 - Categorização dos benefícios das atividades físicas citados pelos enfermeiros (São Paulo, 2000)

\begin{tabular}{|l|c|}
\hline \multicolumn{1}{|c|}{ BENEFÍCIOS } & Citações \\
\hline Físicos & \\
Estimular a circulação, a agilidade, a força & \\
Permitir avaliação da psicomotricidade e melhorar & 2 \\
algum comprometimento motor & \\
Manter o condicionamento físico do paciente & 1 \\
Possibilitar sensação de bem-estar físico & 2 \\
Possibilitar diminuição do sedentarismo & 1 \\
Permitir a movimentação do paciente & 1 \\
Permitir que o paciente fique mais ativo & 1 \\
Possibilitar gasto de energia & 1 \\
\hline Psíquicos & 2 \\
Diminuir a ansiedade; descarregar tensões & \\
Melhorar a disposição para as atividades (conversar, & 2 \\
tomar banho, etc) & 1 \\
Possibilitar sensação de bem-estar psíquico & 1 \\
Permitir momento de lazer, de relaxamento & 2 \\
Trazer de volta para a realidade & 1 \\
Possibilitar a expressão de sentimentos & 1 \\
Estimular pacientes deprimidos & 1 \\
"Tirar" o paciente do quadro de agitação & 1 \\
Permitir que fique mais ativo psiquicamente & 1 \\
Possibilitar a avaliação da orientação espacial do paciente & 1 \\
Ajudar paciente negativista a ter iniciativa & \\
Melhorar a auto-estima do paciente & \\
\hline Social & \\
Estimular a cooperação entre os pacientes & \\
Permitir a socialização & \\
Melhorar o relacionamento & \\
"Tira" o paciente do leito & \\
\hline & \\
\hline
\end{tabular}

Estudos apontam que há uma dificuldade dos profissionais de saúde mental considerar mente-corpo em suas intervenções ${ }^{(7)}$.

"Trazer o paciente para a realidade" constitui-se num benefício psíquico apontado, bem como "Possibilitar a expressão de sentimentos" e alguns relatos são apresentados a seguir, para melhor ilustrar tais constatações.

Acho que faz bem, traz de volta uma realidade, acho que um lado saudável da vida que fica esquecido...

(...) Se eles estão com raiva de alguma coisa eles vão botar pra fora. Nos jogos eles comentam, eles fazem comentários, eles botam pra fora as coisas que eles querem falar. Não é aquela coisa assim de sentar e conversar, né! Jogando eles se soltam e vão falando, né. Tudo o quê eles têm vontade!.
"Diminuir a ansiedade; descarregar tensões", outro benefício psíquico referido é apresentado na fala seguinte.

\section{(...) Diminuir a ansiedade, bastante, isso é uma das principais características que nós percebemos. Os pacientes ficam bem mais tranqüilos, eles descarregam as tensões quando eles estão jogando bola, quando estão jogando basquete, fazendo ginásti- ca e no grupo de expressão também.}

Outrossim, "Permitir a socialização" foi o benefício mais citado (6). Alguns relatos dos profissionais explicitam tal fato.

Primeiro que é assim, facilita a socialização porque é legal para eles estarem numa atividade junto e tal...

Porque tem pacientes que são muitos isolados, né! Uns não querem fazer nada e se deixar eles ficam o tempo todo no leito, então esse grupo(...) o objetivo dele é justamente esse: que haja uma interação entre eles.
Fatores que influenciam os enfermeiros a utilizarem a atividade física na assistência a pacientes psiquiátricos 


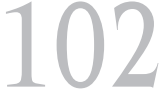

Rev Esc Enferm USP 2003; 37(3): 97-105.
$\mathrm{Na}$ literatura clássica sobre assistência de enfermagem ao paciente esquizofrênico, alheado da realidade, três são os focos gerais para a intervenção de enfermagem: atender as necessidades básicas, facilitar a comunicação e facilitar a participação social ${ }^{(12)}$.

Usar os exercícios físicos para alcançar o objetivo de socializar o paciente e melhorar sua comunicação pode ser uma boa estratégia de intervenção de enfermagem.

A maioria das enfermeiras entrevistadas aponta como fatores facilitadores para a promoção de atividades físicas, a disposição do paciente, a disponibilidade da equipe multidisciplinar e o espaço físico adequado.

No tocante às dificuldades para a promoção de atividades físicas, o número reduzido de funcionários é citação dominante, seguido de falta de espaço físico adequado.

Alguns desses resultados podem ser vistos no trabalho de Mckenna et al. ${ }^{(10)}$, no qual as enfermeiras citam como as principais barreiras: falta de tempo, falta de sucesso, falta de incentivo, falta de pesquisa, falta de protocolo.

Quanto ao espaço físico inadequado, lembramos que o hospital conta com duas quadras, uma interna e outra externa; mesmo assim, este problema é referenciado. O hospital conta com quatro enfermarias, sendo que todos os pacientes das mesmas, de forma não sistematizada usam estas quadras o que pode dificultar o seu melhor aproveitamento. Assim sendo, observamos ser necessária uma rotina indicando dia e hora disponíveis para que os pacientes de cada enfermaria façam uso das quadras. Notamos a importância da atividade física tornar-se parte da rotina da instituição, solucionando assim alguns tipos de problemas.

No que se refere ao diagnóstico do nível de atividades físicas destes enfermeiros, baseado no questionário de Matsudo ${ }^{(9)}$, notamos que a maioria deles está no estágio de "AÇÃO", 7 enfermeiros (50\%), 6(42,9\%) são "CONTEMPLATIVOS", e apenas 1 enfermeiro $(7,1 \%)$ está na fase "PRÉCONTEMPLATIVA"

Vale a pena lembrarmos que no estágio de “PRÉ-CONTEMPLAÇÃO" o indivíduo não tem intenção de mudar seu comportamento e o que está na fase de CONTEMPLAÇÃO con- sidera seriamente a possibilidade de mudança. Já o indivíduo na fase de AÇÃO está muito empenhado em mudar e já mostra ações concretas.

De acordo com os dados apresentados, verificamos que dos 7 enfermeiros que estão no estágio “AÇÃO”, dois deles não desenvolvem atividades com os pacientes, sendo que um desempenha apenas atividades administrativas e, no seu cotidiano, não tem oportunidade de dar cuidados diretos aos pacientes.

O mesmo acontece com os 6 enfermeiros da fase de "CONTEMPLAÇÃO", dos quais 2 também não oferecem atividades, mas são enfermeiros assistenciais.

\section{CONSIDERAÇÕES FINAIS}

Hospitais psiquiátricos estão freqüentemente lotados de pessoas que parecem ter perdido o interesse pela realidade, de ter perdido o senso de valor pessoal e que parecem estar indiferentes às outras pessoas com quem convivem diariamente.

A interação grupal é um dos modos mais bem sucedidos para estimular essas pessoas a recuperar a auto-estima, seu interesse pelo seu ambiente e pela sua vida cotidiana ${ }^{(11)}$.

As atividades físicas, exercícios físicos, desenvolvidos em grupo com os pacientes, podem se constituir, então, em ótima estratégia grupal utilizada pelo pessoal de enfermagem.

Muitos estudos têm indicado que a atividade física regular está positivamente associada com saúde mental e social, além de prevenir fatores de risco coronarianos, pois melhora estilo de vida e socialização.

Mckenna et al. ${ }^{(10)}$ mostraram, em seu estudo, os efeitos poderosos que os comportamentos individuais dos profissionais da saúde podem ter sobre seu comportamento profissional; o principal achado é que aqueles profissionais em estágio de "AÇÃO" ou de "MANUTENÇÃO" de mudança de sua própria atividade física, promovem 3 vezes mais atividades físicas com seus pacientes do que aqueles em outros estágios.

A idéia básica transmitida pelo autor é de que profissionais de saúde que têm um modo de vida particular, por exemplo, que se exercitam regularmente, transferem suas crenças, atitudes e comportamentos para seus pacientes. 
Por outro lado, estudos mostram a importância de rotinas acerca das atividades físicas serem amplamente institucionalizadas para encorajar altos níveis de promoção de atividades saudáveis ${ }^{(4,10)}$.

Burns $^{(3)}$ reforça recomendações acerca da atividade física como um meio de promover saúde. Estratégias chave para manutenção da rotina de atividade física, recomendadas pelo autor podem, no nosso entender, ser extrapoladas para a situação hospitalar, considerando que os pacientes retornam para seus lares. A idéia básica é: estabelecer uma rotina de atividades, permitir que os exercícios sejam prazerosos, monitorar os progressos alcançados pelo próprio cliente e estabe- lecer um sistema de documentação que registre, não só os progressos, como sirva de motivação.

Finalizando, gostaríamos de salientar que a idéia de promover atividades físicas junto a pacientes psiquiátricos deve constituir-se em estratégia de cuidado de enfermagem que supere o cuidado meramente técnico e se amplie para um cuidado dito subjetivo. Outrossim, deve também refletir as diretrizes dos processos de mudança da atenção em saúde mental em curso, em nosso país, cuja causa principal é a de gerar cuidado e assistência em saúde mental, respeitando as diferenças, os direitos de cidadania e, acima de tudo, a liberdade.

\section{REFERÊNCIAS}

(1) Pratt M. Benefits of lifestyle activity vs structured exercise. JAMA 1999; 281(4): 375-6.

(2) Karnoven MJ. Physical activity for a healthy life. Res Q Exerc Sport 1996;67(2):213-5.

(3) Burns KJ. A new recommendation for physical activity as a means of health promotion. Nurse Pract 1996; 21(9): 18, 21-2,26-8.

(4) McDowell N, McKenna J, Naylor PJ. Factors that influence practice nurses to promote physical activity. $\mathrm{Br} \mathrm{J}$ Sports Med 1997;31:308-13.

(5) Manenti SA., Ciamponi MHT Esportes e reabilitação: melhoria da qualidade de vida dos membros da Associação Esportiva da Divisão de Medicina de Reabilitação do HCFMUSP.[monografia].São Paulo(SP): Escola de Enfermagem da USP; 1997.

(6) Dunninghanm W, Aguiar WM. O custo social dos transtornos mentais. J Bras Psiq 1995; 44(8): 419-22.
(7) Beesley S, Mutrie N. Exercise is beneficial adjunctive in depression. [letter]. BMJ 1997; 315(6): 1542-3.

(8) Broocks A, Meyer TF, George A, Pekrunn G, Hillmer-Vogel U, Hajak G, et al. Exercise avoidance and impaired endurance capacity in patients with panic disorder. Neuropsychobiology 1997; 36(4): 182-7.

(9) Secretaria do Estado da Educação. Secretaria do Estado da Saúde. Centro de Estudos do Laboratório de Aptidão Física de São Caetano do Sul. O trabalho educacional na adesão ao exercício físico. São Paulo; 1997.

(10) McKenna J, Naylor PJ, Mcdowell N. Barriers to physical activity promotion by general practioners and practice nurses. Br J Sports Med 1998; 32(4): 242-7.

(11)Taylor CM. Mereness's essentials of psychiatric nursing. $11^{\mathrm{a}}$ ed. St. Louis: Mosby; 1982.

(12) Stuart GV, Sundeen SJ. Principles and practice of psychiatric nursing. $40^{\mathrm{a}}$ ed. St. Louis: Mosby; 1991.
Fatores que influenciam os enfermeiros a utilizarem a atividade física na assistência a pacientes psiquiátricos
Recebido: 17/09/2002 Aprovado: 28/07/2003

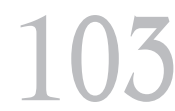

Rev Esc Enferm USP 2003; 37(3):97-105. 


\section{INSTRUMENTOPARACOLETADEDADOS}

Identificação:

Sexo: feminino ( ) masculino ( )

Idade :

Estado civil :

Onde se formou?

Tem pós-graduação? ( ) lato sensu ( ) stricto sensu 1. Mestrado, 2. Doutorado

Há quanto tempo trabalha na área?

Função que exerce:

1. Você desenvolve atividades físicas junto aos pacientes ? ( ) sim ( ) não

2. Como são essas atividades?

3. Você considera que elas possam trazer algum benefícios ao paciente? Quais?
( ) Físico ( ) Psíquico ( ) Outros
Especifique:

4. Você considera que elas possam trazer algum malefício ao paciente? Quais?

( ) Físico ( ) Psíquico ( ) Outros

Especifique:

5. Quais as dificuldades enfrentadas por você, para promover atividades físicas, para os pacientes?

6. Quais são as facilidades enfrentadas por você para promover atividades físicas para os pacientes? 
Questionário para diagnóstico do nível de atividade física da população alvo (Secretaria do Estado da Educação, Matsudo, 1997).

A mudança de comportamento envolve 4 estágios:

1- PRÉ - CONTEMPLAÇÃO: sem intenção de mudança

2- CONTEMPLAÇÃO: considerando seriamente em mudar

3- PREPARAÇÃO: fazendo pequenas mudanças de comportamento

4 AÇÃO: muito empenhado em mudar e já com ações concretas

O estágio de comportamento dos sujeitos pode ser classificado, de uma forma simples, considerando-se as perguntas que seguem.:

Assinale APENAS UMA frase que exprima melhor o que você pensa em relação ao exercício hoje:

(1) Eu penso em me dedicar a fazer exercício regularmente mas nunca consigo

(2) Eu não penso em me dedicar a fazer exercício

(3) Eu gostaria de me dedicar a fazer exercício regular mas não tenho certeza como começar

(4) Eu estou seriamente pensando como me dedicar a fazer exercício regularmente em um futuro próximo

(5) Às vezes eu penso que deveria me dedicar ao exercício regular

(6) Agora eu não quero me dedicar ao exercício regular

(7) Às vezes eu me preocupo com a falta de exercício regular

(8) Eu fiz exercício regular no passado e gostaria de voltar a fazer isso logo

(9) Eu não tenho necessidade de fazer exercício regular

(10) Eu já faço exercício e quero manter

(11) Eu faço exercício e quero aumentar

Considerando as respostas obtidas os sujeito poderão ser classificados assim:

Pré- Contemplativo: Alternativas 2,6 e 9

Contemplativo: Alternativas 1,3,4,5,7 e 8

Ativo: Alternativas 10 e 11
Fatores que influenciam

os enfermeiros a

utilizarem a atividade

física na assistência a

pacientes psiquiátricos 